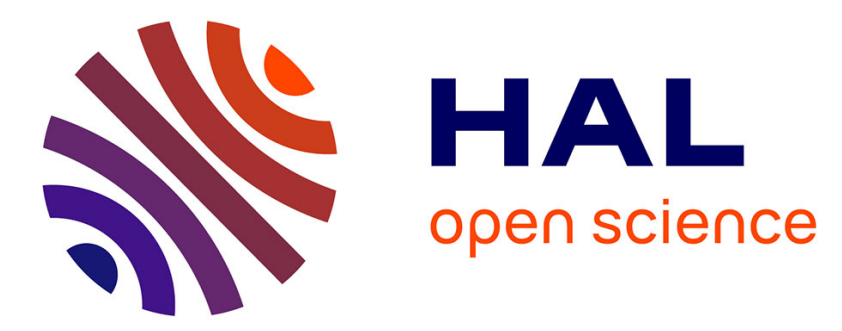

\title{
Evidence for the predominant influence of the asymmetry degree of freedom on the evenodd structure in fission-fragment yields
}

Manuel Caamaño, F. Rejmund, Karl-Heinz Schmidt

\section{- To cite this version:}

Manuel Caamaño, F. Rejmund, Karl-Heinz Schmidt. Evidence for the predominant influence of the asymmetry degree of freedom on the evenodd structure in fission-fragment yields. Journal of Physics G: Nuclear and Particle Physics, 2011, 38 (3), pp.35101. 10.1088/0954-3899/38/3/035101 . hal00600884

\section{HAL Id: hal-00600884 \\ https://hal.science/hal-00600884}

Submitted on 16 Jun 2011

HAL is a multi-disciplinary open access archive for the deposit and dissemination of scientific research documents, whether they are published or not. The documents may come from teaching and research institutions in France or abroad, or from public or private research centers.
L'archive ouverte pluridisciplinaire $\mathbf{H A L}$, est destinée au dépôt et à la diffusion de documents scientifiques de niveau recherche, publiés ou non, émanant des établissements d'enseignement et de recherche français ou étrangers, des laboratoires publics ou privés. 


\title{
Evidence for the predominant influence of the asymmetry degree of freedom on the even-odd structure in fission-fragment yields
}

\author{
Manuel Caamaño ${ }^{1} \ddagger$, Fanny Rejmund ${ }^{1}$ and \\ Karl-Heinz Schmidt ${ }^{1,2}$ \\ ${ }^{1}$ GANIL, CNRS/IN2P3, CEA/DSM, bd Henri Becquerel, 14076 Caen \\ ${ }^{2}$ GSI, Planckstrasse 1, 64291 Darmstadt, Germany \\ E-mail: frejmund@ganil.fr
}

\begin{abstract}
Based on a comprehensive set of fission-fragment distributions measured in low-energy fission, the even-odd staggering in the fission-fragment element yields is investigated. The well-established evolution of the global even-odd effect with the fissioning system is found to be only a partial aspect of the even-odd structure. Indeed, it is shown that the global even-odd effect varies systematically with the mean asymmetry of the fission-fragment distribution, and that the general increase of the even-odd staggering with asymmetry depends on the fissioning system. Thus, the dependency of the even-odd effect with the fissioning system is accredited in part to the asymmetry evolution of the charge distribution, and not solely to the dissipated energy as it has been done earlier. This interpretation is strongly supported by data measured in inverse kinematics, which cover the complete fission-fragment charge distribution and include precise yields at symmetry. The relevance of the ordering parameter for the description the even-odd effect in fission-fragment yields as a general property is explored.
\end{abstract}

PACS numbers: 24.75.+i, 25.70.-z, 25.85.-w, 25.85.Ec, 25.85.Jg

Submitted to: J. Phys. G: Nucl. Phys.

\section{Introduction}

Even-odd staggering in proton $(\mathrm{Z})$ and neutron $(\mathrm{N})$ numbers is a well-known characteristic of low-energy fission-fragment yields [1]. The large amplitude of this staggering, which may reach $40 \%$ in the case of the element distribution of fission fragments of thorium [2], has always been fascinating to nuclear physicists. The experimental access to the exact number of neutrons of the fission fragments at scission is difficult to obtain because the number of neutrons is modified by evaporation processes. In contrast, evaporation of protons from the neutron-rich fission fragments is negligible.

$\ddagger$ Present address: Universidade de Santiago de Compostela, E-15706 Santiago de Compostela, Spain 
Therefore, if measured prior to their beta-decay, the element yields, and the associated even-odd staggering, reveal the properties of the fissioning system at scission. During the fission process, the total energy of the system is subject to substantial reorganization. At the fission barrier, the part of the initial excitation energy which corresponds to the height of the barrier is bound in potential energy and the intrinsic excitation energy is accordingly reduced. On the further path to scission where the two fragments separate, the potential energy decreases again. This energy release drives the nascent fragments apart and feeds the pre-scission kinetic energy.

The observation of odd-Z fragments from an even-Z fissioning nucleus testifies to the breaking of proton pairs during the re-organisation of the intrinsic structure of the fissioning nucleus on its path towards scission. Indeed, in thermal-neutron-induced fission of even-Z actinides, the intrinsic energy available above the fission barrier is lower than the pairing gap, and thus, the system crosses the fission barrier in a fully paired configuration. When odd-Z fragments are observed, an ensemble of fully paired protons undergoes deformation down to scission, where at least one proton pair is broken and both unpaired protons end up in different fission fragments, producing odd-Z species. The breaking of pairs consumes part of the energy stored in the system in the form of collective kinetic energy [3]. This coupling between collective and individual degrees of freedom is the consequence of the viscosity of the nuclear matter, and is defined as a dissipation process. The amplitude of the even-odd staggering is therefore linked to dissipation during the deformation in the fission process. It has always been a challenge to understand the mechanism of dissipation and the relation between pairing effects and the dissipated energy. The present work focuses on isotopes for which nuclear-charge yields are accessible. The data emerge from experiments performed at ILL for fissile actinides and at GSI for long chains of neutron-deficient actinium, thorium, protactinium, and uranium isotopes. This ensemble of data allows accessing an unprecedented systematic on pairing effects in fission-fragment yields. They show new general properties that cannot be explained with the conventional interpretations.

\section{Global even-odd staggering and dissipated energy}

A simple method to quantify the even-odd structure in the fragment production is to define the global even-odd effect $\delta$ as the average difference between even- and odd-Z total yields over the full available distribution [2]:

$$
\delta=\frac{\sum_{Z \text { even }} Y(Z)-\sum_{Z \text { odd }} Y(Z)}{\sum_{Z} Y(Z)}
$$

Several models have attempted to quantitatively relate the global even-odd staggering to the dissipated energy. They are all based on the idea that the evenodd effect in element yields is determined by the probability that the protons remain in fully paired all the way up to the scission configuration. 
The model proposed by Nifenecker et al. [4] is certainly the most widely used as it correlates $\delta$ and the dissipated energy $E_{\text {diss }}$ with a very simple expression. This model calculates the maximum number of broken pairs at scission as the ratio between the dissipated energy and the amount of energy necessary to break a pair (the pairing gap). The nucleus at scission is then considered to consist of a fully paired core and an ensemble of broken pairs. The probability to break a proton versus a neutron pair, the probability to break one or more pairs if the necessary amount of energy is available, and the probability that the nucleons of the broken pairs end up in two different fragments, are determined by a combinatorial analysis. This model estimates a dissipated energy of about $4 \mathrm{MeV}$ for ${ }^{230} \mathrm{Th}$ to $12 \mathrm{MeV}$ for ${ }^{250} \mathrm{Cf}$.

An alternative approach by Bouzid et al. [5] is based on a dynamical description of the fission process. In this model, the descent from saddle to scission is considered to be adiabatic, and the violent neck rupture leading to the formation of the two separated fragments causes the pair breaking. The probability to break a pair is correlated with the velocity of the neck rupture, which is shown to increase with the Coulomb repulsion at the scission point. The Coulomb repulsion is linked to the Coulomb parameter $Z_{c}=Z_{f}^{2} / A_{f}^{1 / 3}$, where $Z_{f}$ and $A_{f}$ are the nuclear charge and mass of the fissioning nucleus, respectively. The probability that the broken pairs end up in different fragments is a parameter fitted to reproduce the data. In particular, this model considers the difference in the proton- and neutron-number staggering as a consequence of the less violent neck rupture for protons, since they are less likely to be present in the neck due to the Coulomb repulsion.

A rigorous formulation of the even-odd staggering has been derived by Rejmund et al. [6] within the framework of the statistical model. It is based on a realistic description of the number of quasi-particle excitations of the proton and neutron ensembles as a function of the excitation energy at scission. The pairing-gap parameter depends on excitation energy, deformation, and the number of quasi-particle excitations. The probability to break proton pairs as a function of the excitation energy is derived accurately as the ratio of the proton excited states over all available single-particle states. This is in contrast to the model of Nifenecker et al., where the even-odd effect is deduced from a combinatorial analysis, based on the ensemble of possibly broken pairs, using fitted parameters.

Recently, new ideas about the division of the intrinsic excitation energy between the two nascent fragments [7] lead to an extension of the model of Rejmund et al. [6] to asymmetric splits. The development of this new model [8] was guided by the analysis of the empirical data performed in the present work. The central idea is that the magnitude of the even-odd effect is not governed by the total intrinsic energy available in the fissioning system but by the nascent fragment which carries the lowest intrinsic excitation energy at scission.

The evolution of the global even-odd staggering from measurements of fragment yields of fissile actinides at ILL is displayed as a function both of the fissility and the Coulomb parameter in figure 1, left and right panels, respectively. A clear decrease is 

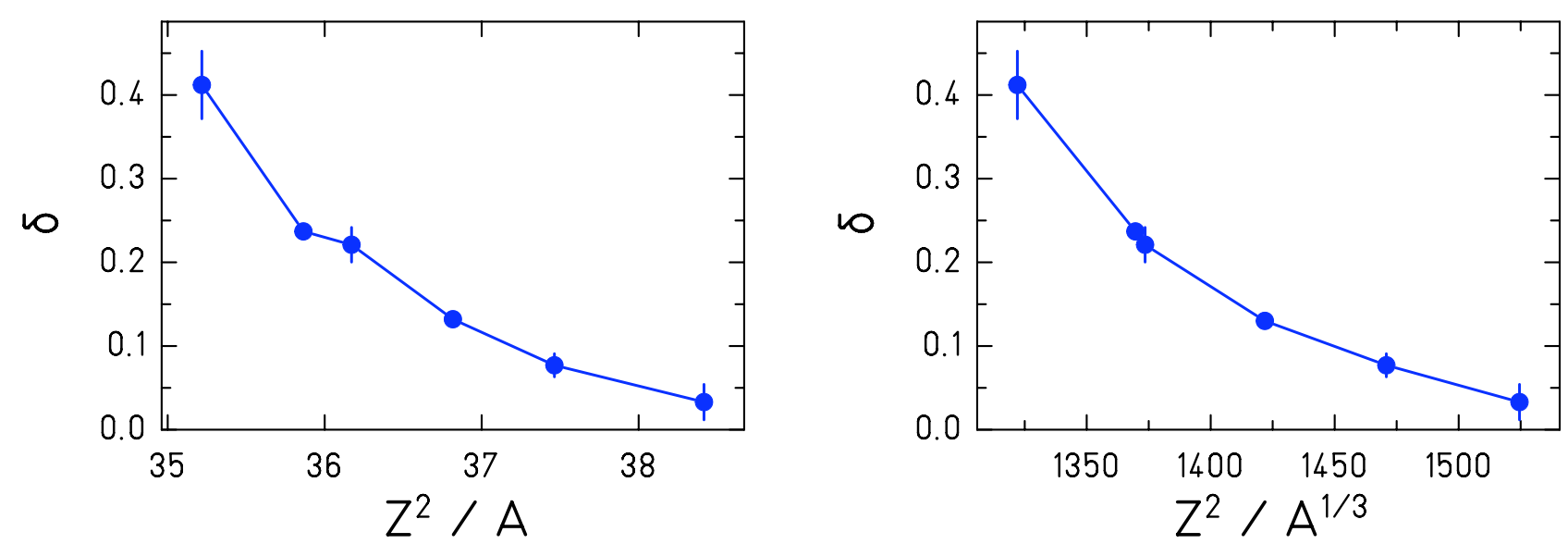

Figure 1. Global even-odd effect as a function of the fissility parameter (left) or the Coulomb parameter (right). Data are from thermal-neutron-induced fission of ${ }^{229} \mathrm{Th}$ $[2],{ }^{233} \mathrm{U}[14],{ }^{235} \mathrm{U}[3,15],{ }^{239} \mathrm{Pu}[16],{ }^{245} \mathrm{Cm}[17,18]$ and ${ }^{249} \mathrm{Cf}[19,20]$.

observed as a function of both parameters. The correlation of the even-odd staggering with the fissility [9] suggested that more energy is dissipated in nuclei with higher fissility, since the amplitude of the global even-odd effect was already associated with the dissipated energy gained by the nucleus. The fissility parameter $x=Z_{f}^{2} / A_{f}$ reflects the stability against fission, but its connection with the dissipated energy is not evident. On the other hand, the total energy released from saddle to scission has been shown to be essentially determined by the Coulomb parameter [5]. Therefore, the evolution of the even-odd staggering with the fissioning nucleus is usually investigated as a function of the Coulomb parameter $[6,10,11]$.

\section{Evolution of the fragment distribution with the fissioning nucleus and its influence on the even-odd staggering}

In low-energy fission of most actinides, shell effects induce an asymmetry in the mass and charge distributions of fragments, which show two groups [12]. The group of heavy fragments is distributed around an average value of $\mathrm{A} \sim 140$, independent of the mass of the fissioning system. In order to conserve the total mass of the fissioning nucleus, the group of the light fragments increases towards heavier masses for heavier fissioning nuclei, gradually approaching symmetry. The equivalent behaviour is observed in charge distributions. However, for the latter, until an experiment exploiting inverse kinematics [13], only the light part of the distribution was experimentally accessible. Element-yield distributions reported in figure 2 have been obtained at the Lohengrin spectrometer $[2,14,3,15,16,17,18,19,20]$. For this particular experimental procedure, only the light fragment distributions were measured. In addition, the present analysis uses solely those element yields for which the complete isotopic distribution is available. This restriction is 
necessary to avoid incorrect element yields [21]. The data show an average light charge centred on values varying from $\mathrm{Z}=36$ to 44, for fissioning nuclei from $\mathrm{Th}$ to $\mathrm{Cf}$, as indicated by arrows in figure 4 . This coincides with a heavy charge distribution centred on a constant value $\mathrm{Z}=54$, independent of the fissioning nucleus, as reported already in [22]. To explore the fission asymmetry, it is convenient to define the asymmetry parameter: $a=\left(Z_{H}-Z_{L}\right) / Z_{f}$, where $Z_{H}$ and $Z_{L}$ are the charge of heavy and light fragment, respectively. In figure 2 , the element yields are displayed as a function of this parameter, which results in a common scale for the asymmetry of the distribution for all considered systems. As it was previously mentioned, and in order to conserve the total nuclear charge, the average asymmetry $\langle a>$ of the nuclear-charge distributions of the light fission-fragments shifts from 0.28 to 0.13 when considering fissioning systems from ${ }^{230}$ Th to ${ }^{250} \mathrm{Cf}$. In figure 3 , the corresponding global even-odd effect is displayed as a function of the average asymmetry of the light fragment distribution. A clear relationship is observed; when the average asymmetry decreases, the global even-odd effect decreases.

As there exists a clear decrease of the even-odd effect with increasing excitation energy at saddle $[23,24]$ and a prediction for an increasing energy release between saddle and scission with the Coulomb parameter $[5,10]$, it is conventionally assumed that the decrease in even-odd effect with the fissility or with the Coulomb parameter is due to the increase in dissipated energy from saddle to scission. However, figures 1 and 3 show that the global even-odd effect is correlated also with other parameters which are not directly connected to the energy dissipated between saddle and scission; the fissility $x$ and the average asymmetry $\langle a>$ of the fragment distribution. The clear correlation between the global even-odd effect and the mean asymmetry opens the question on the potential influence of the asymmetry degree of freedom on the even-odd effect.

\subsection{Local even-odd staggering and influence of the asymmetry}

In order to obtain more detailed information on the influence of the asymmetry degree of freedom on the amplitude of the even-odd effect, the local even-odd staggering $\delta(Z)$ can be investigated. Its magnitude is a measure of the deviation of the nuclear-charge distribution from a smooth behaviour, and is usually studied following the prescription of Tracy et al. [25]. For different fissioning systems [2, 14, 3, 15, 16, 17, 18, 19, 20], the local even-odd staggering has been shown to be larger for large asymmetry. This experimental observation is presented in figure 4 for a number of nuclei. The rate of

increase is largest for the lightest system, ${ }^{230} \mathrm{Th}$, and decreases for the heavier systems. The values of the local even-odd effect for the different nuclei approach each other at symmetry, but due to missing experimental information at symmetry and due to the scattering of the data points one can only say that they converge to a rather narrow range between 5 and 20 percent. For several systems, the data points that are closest to symmetry present an appreciably higher local even-odd effect than expected from the global trend. This effect may be associated with the influence of the $\mathrm{Z}=50$ shell 


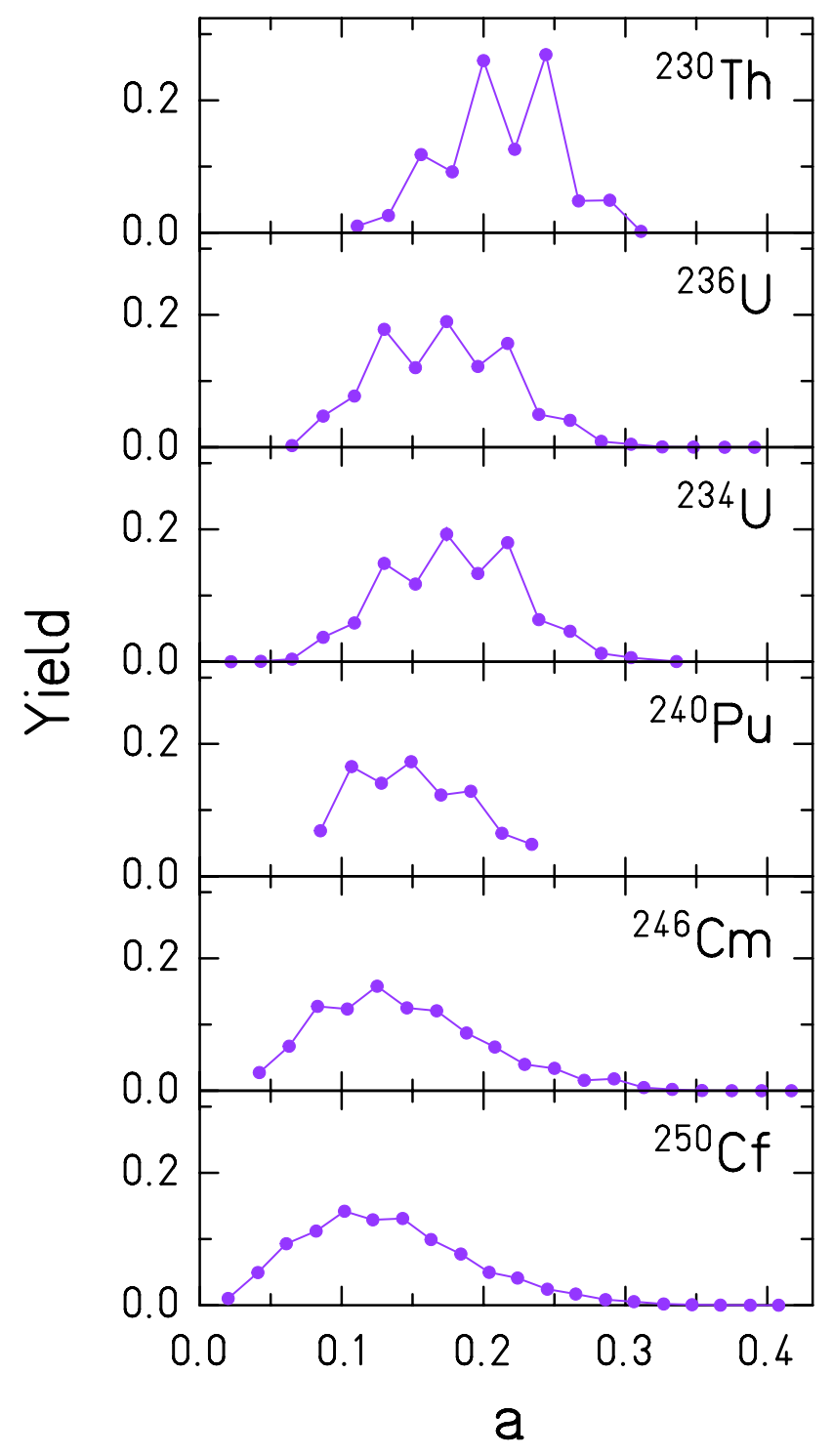

Figure 2. Element distributions displayed as a function of the asymmetry parameter of the distribution, for thermal-neutron-induced fission of ${ }^{230} \mathrm{Th},{ }^{236} \mathrm{U},{ }^{234} \mathrm{U},{ }^{240} \mathrm{Pu}$, ${ }^{246} \mathrm{Cm}$ and ${ }^{250} \mathrm{Cf}$, from top to bottom.

in the complementary fragment, which is known to enhance the yield of tin isotopes and, thus, leads to a local increase of the deduced even-odd effect. The local even-odd effect, namely the increase towards asymmetry has led to the notion of cold asymmetric fission, for which an extreme deformation takes most of the available excitation energy, and consequently the intrinsic excitation energy would remain low [15]. However, no elaborate model existed to quantitatively describe the even-odd effect based on these assumptions. Moreover, it will be shown in the following that, even in the case of scission configurations with invariably at least one unpaired proton, a large even-odd 


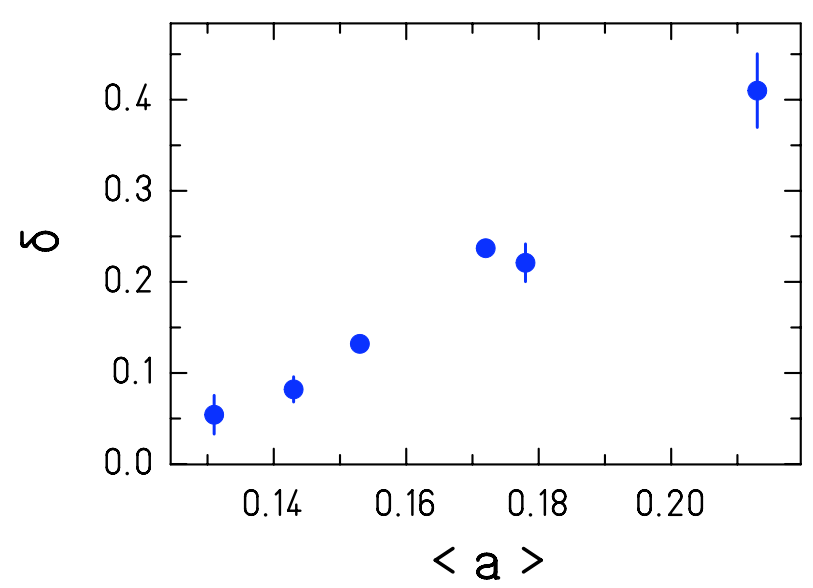

Figure 3. Global even-odd effect as a function of the average asymmetry of the fragment distribution.

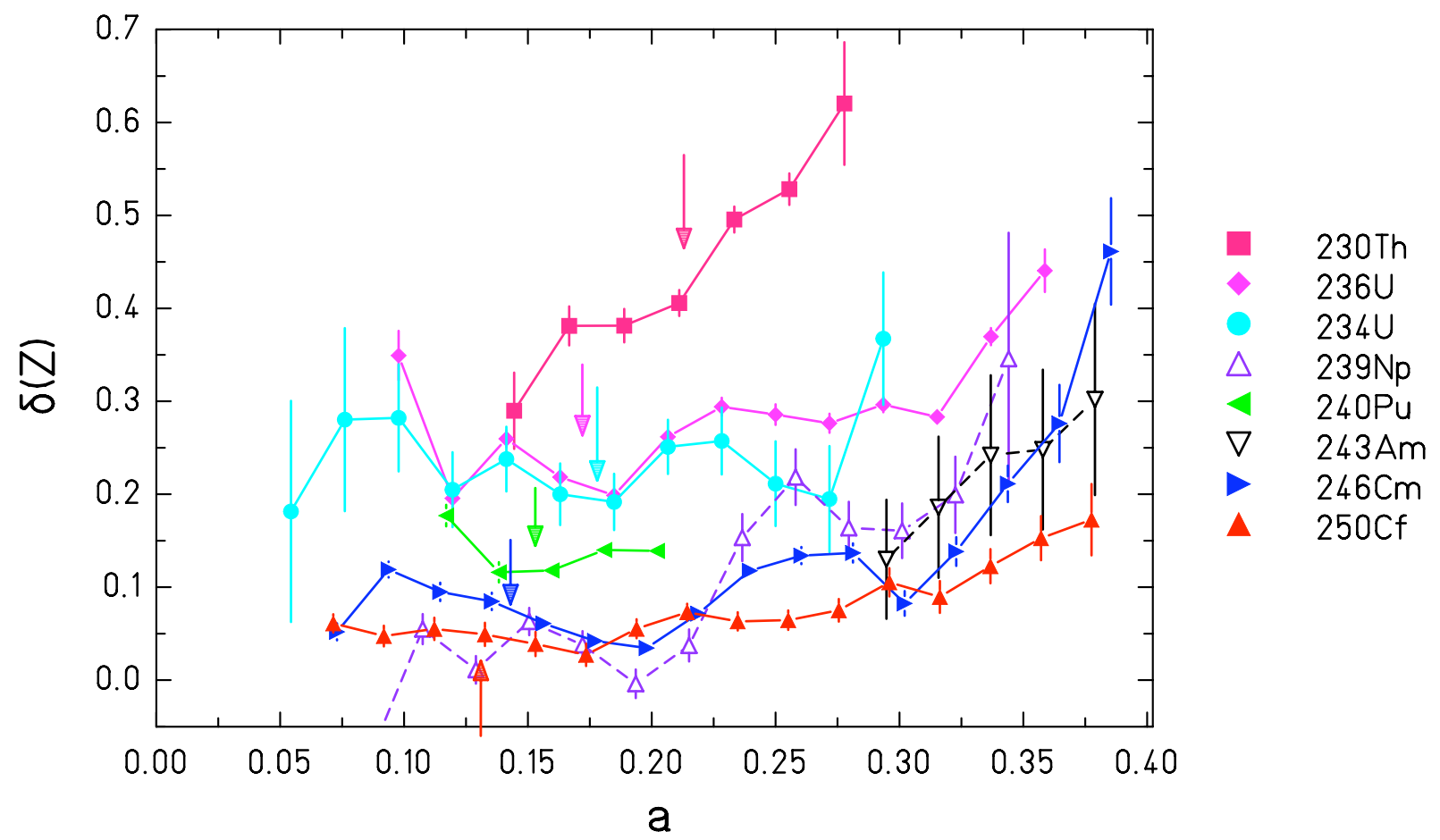

Figure 4. Local even-odd effect for the series of element distributions of even$[2,14,3,15,16,17,18,19,20]$ and odd-Z $[26,27]$ nuclei studied with neutron-induced fission. The arrows indicate the value of the average asymmetry of the fragment distributions. 
effect can be observed at large asymmetry. Therefore, the excitation energy and the consequent number of broken pairs cannot be considered as being uniquely accountable for the amplitude of the even-odd effect.

\subsection{Local even-odd staggering in odd-Z fissioning systems}

In the late 90 's the general appearance of a large even-odd effect in the $\mathrm{Z}$ distributions of odd-Z fissioning nuclei has been reported [28]. In these odd-Z systems, the probability to reach the scission configuration with at least one unpaired proton is always one. Assuming that any unpaired proton ends up in one or the other fragment with equal probability, it was expected that the even-odd effect would be zero over the full element distribution. With experimental techniques based on inverse kinematics, the evenodd staggering has been systematically measured for a large number of actinium and protactinium isotopes, over the complete fragment distribution. Its value was found to be zero close to symmetry and systematically increasing for large asymmetry, up to amplitudes as large as $40 \%$. A similar observation of a large even-odd effect has been reported in neutron-induced fission of ${ }^{238} \mathrm{~Np}$ [26], and ${ }^{242 m} \mathrm{Am}$ [27], as shown in figure 4. The even-odd effect of the odd-Z fissioning systems at large asymmetry follows the general behaviour of the even-Z fissioning systems. Their values are very close to those of the neighbouring even-Z systems, as was reported already in [26]. In the inversekinematics data, where the heavier part of the fragment distribution has been measured, the even-odd staggering was found to be negative for heavy fragments, revealing a higher probability for the unpaired proton to end up in the heavy fragment. In these odd-Z fissioning systems, an even-odd effect may only be generated by a mechanism which is related to some difference in the properties of the fragments in asymmetric splits. The similarity of the local even-odd effect values of close-by even-Z and odd-Z systems suggests that a similar mechanism is responsible for the increase of the even-odd effect in even-Z fissioning systems. These experimental observations lead to the interpretation that the conventionally assumed relation between pair breaking and even-odd effect in the fission yields is valid only close to symmetry, as it was previously discussed in [28, 6]. In addition, the variation of the even-odd effect close to symmetry is limited to a value below 20\%, which does not vary much as the fissility (or Coulomb parameter) of the fissioning system varies. Most of the amplitude of the even-odd effect is then linked to the asymmetry of the fragment distribution, and is common for odd- $\mathrm{Z}$ and even- $\mathrm{Z}$ fissioning systems.

The observation of an even-odd staggering for odd-Z fissioning nuclei and the interpretation presented reveal that the relation between the amplitude of the even-odd staggering in fission-fragment charge yields and the intrinsic excitation energy at scission is not as direct as suggested by the established models discussed in section 2. Indeed, neither the statistical description of Nifenecker et al. nor the dynamical description of Bouzid et al. can explain the appearance of any global or local even-odd structure for odd-Z fissioning nuclei since in these models, the probability of unpaired nucleons to 
end up in one or the other fragment does not depend on the size of the fragments. The statistical model of Rejmund et al. [6] considers the local effect of dissipated energy for symmetric scission, separately of the influence of asymmetry in the fission-fragment yields. In references [26, 27], the phenomenon is qualitatively discussed in terms of energy balance; because the pairing gap is decreasing with the fragment mass, the light fragments are more likely to remain fully paired. However, only the binding energies of cold systems are considered. A statistical description of the local even-odd staggering as function of the asymmetry based on the level density of the fission fragments formed at scission [28] reproduces the higher probability for the unpaired nucleons to end up in the heavy fragment. This model gives a quantitative prediction of the general increase of the local even-odd staggering with the asymmetry, for odd-Z fissioning nuclei as well as for even-Z fissioning nuclei. Recently, the large local even-odd effect in asymmetric splits has been explained as the result of the energy-sorting mechanism in superfluid nuclear dynamics [8]. This model is the first one that reproduces all experimental data on the local even-odd effect for different fissioning systems and its variation with asymmetry rather well.

\subsection{Local even-odd staggering at symmetry}

The general trend of the even-odd effect towards symmetry is to decrease and to remain restricted to the range between 5 and $20 \%$, as depicted in figure 4 . However, due to the lack of data close to symmetry, it is difficult to draw further conclusions. The experimental technique based on inverse kinematics [13] allows to access the element yields over the complete element distribution, with high resolution and for a wide systematic investigation of neutron-deficient fissioning systems. In addition, fission is induced via electromagnetic interaction, and occurs therefore at excitation energies which are, on the average, a few $\mathrm{MeV}$ higher than in fission induced by thermal neutrons. This results in higher yields for symmetric splits. As a consequence, this technique provides unique and high-quality data on element yields at symmetry. In figure 5, the local even-odd effect measured for similar fissioning systems using both techniques (thermal-neutron-induced fission in direct kinematics and electromagneticinduced fission in inverse kinematics) is displayed in the left panel. The inversekinematics data cover the full element distribution; due to the higher excitation energy the measured even-odd staggering has a lower value, but similar characteristic patterns are observed with both experimental approaches. This is demonstrated in the right panel of figure 5, where the ratio between the amplitude of the even-odd staggering for both experimental methods is displayed. The result is a constant ratio around $60 \%$ on the full range of asymmetry. From this value, it is possible to extrapolate that for both ${ }^{230} \mathrm{Th}$ and ${ }^{234} \mathrm{U}$, the even-odd effect in low-energy fission at symmetry is of the order of few percents, below $10 \%$. It is important to note, that this small but non-zero value of the even-odd staggering at symmetry has two major consequences:

Firstly, the parameterization used for the evaluation of fission-fragment yields [29], 
which is based on former radio-chemical data, considers no even-odd effect at symmetry, in contradiction to the present experimental observation. The parameterization should be reformulated appropriately.
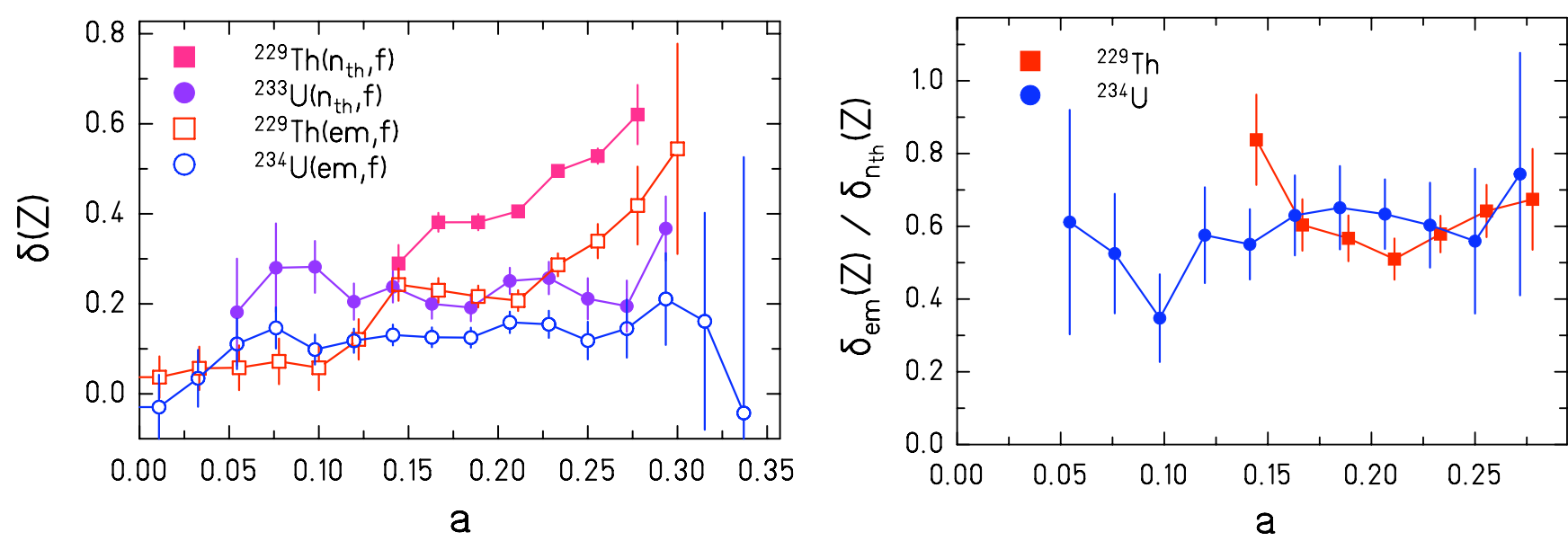

Figure 5. Left panel: Local even-odd staggering measured in similar fissioning systems using thermal-neutron- (full symbols) and electromagnetic-induced- (open symbols) fission. Right panel: Ratio between the data obtained with the two different techniques for the two fissioning systems considered.

Secondly, the major contribution to the global even-odd effect $\left(40 \%\right.$ for ${ }^{230} \mathrm{Th}, 23$ $\%$ for ${ }^{234} \mathrm{U}$, see figure 1) emanates from the asymmetry of the fragment distribution, which is not directly related to the probability to break pairs in the descent from saddle to scission, because it is also present in odd-Z fissioning systems. As a consequence, previous estimates on the dissipated energy derived from the amplitude of the global even-odd staggering $[4,5]$ are not justified. In addition, the estimated value for the evenodd effect at symmetry for ${ }^{230} \mathrm{Th}$ and ${ }^{234} \mathrm{U}$ is very close to the value measured close to symmetry for heavier systems such as ${ }^{246} \mathrm{Cm}$ and ${ }^{250} \mathrm{Cf}$. Both ${ }^{246} \mathrm{Cm}$ and ${ }^{250} \mathrm{Cf}$ have a relatively smooth behaviour when approaching symmetry, suggesting values around few percents (see figure 4). Within the experimental uncertainty, the variation of the amplitude of the even-odd effect at symmetry with the fissility of the fissioning nucleus seems to be weak. The standard interpretation of the global even-odd effect attributes a strong variation of the dissipated energy with the fissility of the fissioning system, based on the evolution of the global even-odd effect with the fissility (figure 1). The present investigation shows the fact that the global even-odd effect is strongly modified by the influence of the asymmetry of the fission process. The even-odd staggering at symmetry remains in a range of small values, and shows at most a weak variation within the experimental error bars. However, the conclusions on the variation of the even-odd effect at symmetry in nuclei studied with neutron-induced fission suffer from the lack of data and large error bars. New data sets and improved measurements would be needed to further precise these observations. 


\subsection{Dependence on asymmetry and fissioning system}

The data analysed in the present work suggest that the local even-odd effect depends on both the fissioning system and the asymmetry of the split. This two-parameter dependence is shown in figure 6, where the local even-odd effect is displayed as a function of the fissility (left panels) and the Coulomb parameter (right panels) of the fissioning nucleus, for different values of the asymmetry (open symbols) and compared to the values closest to or at symmetry (full symbols). The latter are repeated in each figure as reference for better comparison. For symmetric splits measured in electromagneticinduced fission, the local even-odd staggering shows a small but definitively non-zero amplitude, remarkably independent of the fissioning nucleus. The data presented in figure 6 confirm the conclusions drawn from figure 5 for a wider range of nuclei. The data from neutron-induced fission do not provide direct information on this issue, as symmetric splits cannot be reached due to technical limitations already mentioned above. The full squares in figure 6 correspond to yields from the most symmetric splits measured. Due to the properties of the fragment distributions and the technical limitations, discussed in section 3, the access to the most symmetric splits is correlated with the fissioning system, making it easier to experimentally approach the symmetry for larger systems, and decreasing from $a=0.15$ to 0.02 when considering fissioning systems from ${ }^{230} \mathrm{Th}$ to ${ }^{250} \mathrm{Cf}$. This correlation produces a steadily decreasing amplitude of the even-odd effect at the most symmetric yield as a function of fissility or Coulomb parameter.

For large asymmetry (upper panels of figure 6), although fission is induced with moderately higher excitation energy in the inverse-kinematics technique, electromagnetic- and thermal-neutron-induced fission data show the same trend. The amplitude of the even-odd staggering at a given asymmetry is decreasing with fissility or Coulomb parameter. For lower values of the asymmetry (shown in the middle and lower panels of figure 6), the slope and amplitude of the local even-odd effect reduces and gradually approaches the value of the even-odd staggering at symmetry.

\subsection{Ordering parameter}

The dependence of the local even-odd effect with the fissioning system is usually reduced to an ordering parameter defining the fissioning nuclei. An optimum ordering parameter shows the same behavior of the even-odd effect for nuclei with the same parameter. Figure 1 shows that there exists a relation between the global even-odd effect and both the fissility and the Coulomb parameter. The validity of these quantities as ordering parameters for the local even-odd effect is investigated in detail in this section.

Left and right panels of figure 6 show the evolution of the even-odd effect of the

same data set with respect to the fissility and the Coulomb parameter of the fissioning nucleus, respectively. Conventionally $[6,11,10]$, the Coulomb parameter is considered to be the most appropriate ordering parameter, since it is associated with the available energy gain on the path from saddle to scission [5]. However, this conclusion has been 


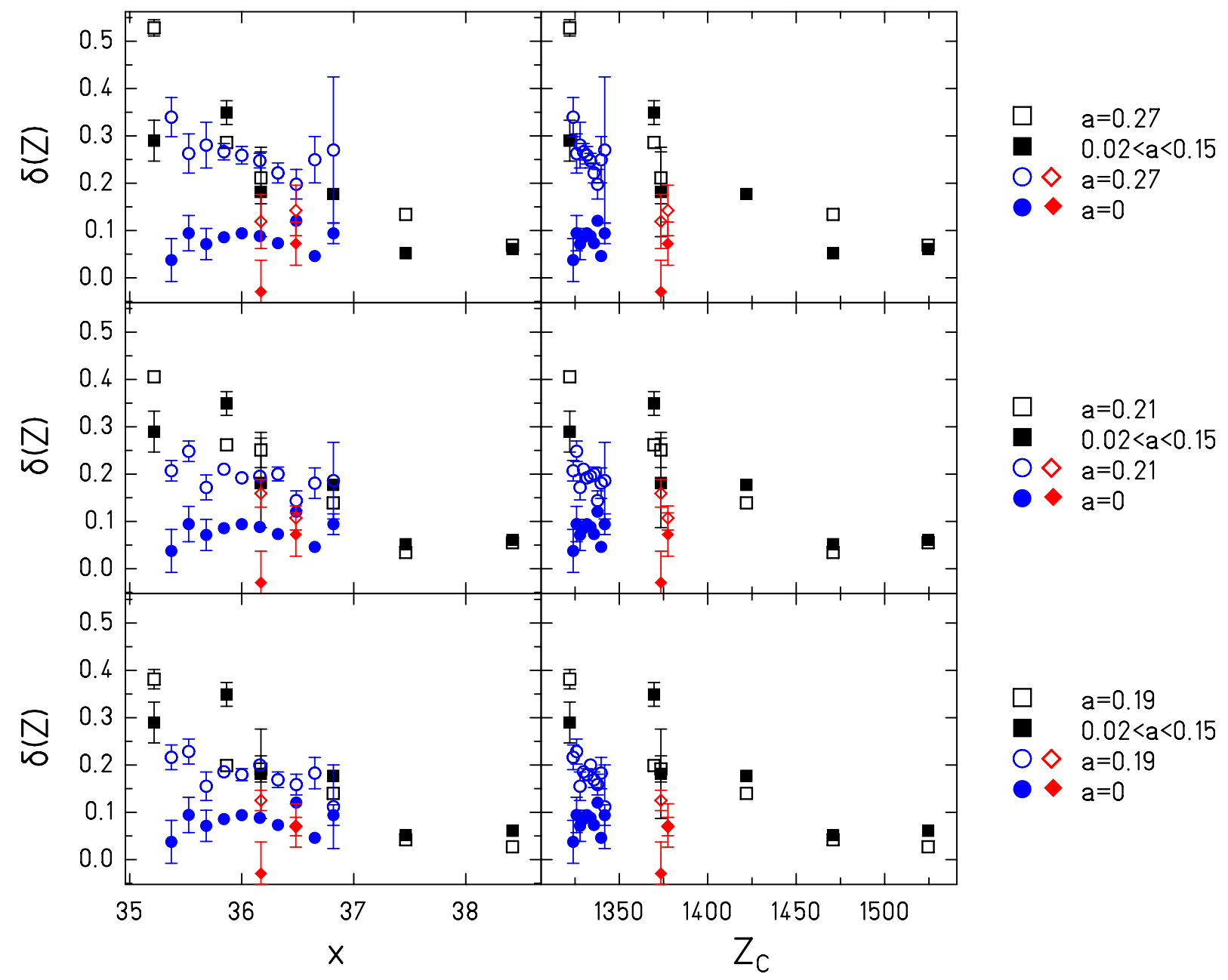

Figure 6. The local even-odd effect is displayed as a function of the fissility $x$ (left panels) and Coulomb parameter $Z_{c}$ (right panels). Upper panels show the effect for an asymmetry of 0.27 (open symbols), and compared to the local even-odd effect measured at symmetry (full symbols). Data of electromagnetic-induced fission of neutron-defficient $\mathrm{Th}$ and $\mathrm{U}$ isotopes are shown as blue circles and red diamonds, respectively. Data from thermal-neutron-induced fission are shown as black squares. Middle and bottom panels: same as above with an asymmetry of 0.21 and 0.19 , respectively. Data at symmetry are shown in all panels for reference.

drawn on the basis of the limited range of accessible nuclei in direct kinematics, while ignoring the correlation between the Coulomb parameter and the average asymmetry of the fragment distribution. Figure 6 displays data for fixed values of the asymmetry distribution, thus eliminating the role of the asymmetry parameter. The panels on the right-hand side show that the data of the long isotopic chains of thorium obtained in electromagnetic-induced fission shrink to a narrow range when plotted as a function of the Coulomb parameter. The systematic decrease of the local even-odd effect with increasing mass number (established in figure 4) produces a steep slope in the even- 
odd effect of each fissioning element when represented as a function of the Coulomb parameter. An extrapolation of the slope in the thorium chain towards higher $Z_{c}$ suggests a zero value of the local even-odd staggering at $Z_{c} \sim 1400$ in the three right panels of figure 6. However, considerable even-odd effects have been observed for heavier systems (see figure 1). Thus, there are severe problems in considering the Coulomb parameter as the ordering parameter for the local even-odd effect, since the behavior of the effect is different when considering different species with the same $Z_{c}$. This could not be observed earlier, because the number of investigated nuclei was too limited (in general only one isotope per fissioning element, as shown by the black squares), and restricted to nuclei close to the stability line. The other possible ordering parameter, the fissility, is investigated in the left panels of figure 6 . This parameter allows for a coherent description of the even-odd effect throughout the whole ensemble of collected data, originating from electromagnetic- or thermal-neutron-induced fission. The systematic decrease of the even-odd effect at fixed asymmetry suggests that the even-odd effect would disappear at $x \sim 39$, which is compatible with the available data on even-odd effect in fission, since all systems investigated have smaller values of fissility.

\section{Influence of the excitation energy}

Figure 5 suggests that an increase in excitation energy influences the even-odd staggering in element yields of the even- $\mathrm{Z}^{234} \mathrm{U}$ and ${ }^{229} \mathrm{Th}$ systems with a constant reduction factor over the complete fragment distribution. The number of initially broken pairs has an impact on the amplitude of the even-odd effect. There exist no experimental data on the evolution of the even-odd effect in element distributions of odd-Z fissioning systems with the excitation energy. However, it is possible to compare neighbouring odd-Z and even-Z systems investigated in electromagnetic-induced fission, where the compound nucleus is created with a few $\mathrm{MeV}$ excitation energy above the fission barrier [13], which allows for the breaking of several nucleon pairs. Interestingly enough, figure 7 shows that, in contrast to the low-energy fission case (figure 4), at several $\mathrm{MeV}$ of excitation energy, the amplitudes of the even-odd staggering in odd-Z fissioning systems are systematically lower than their neighbouring even-Z systems. In low-energy fission, the pairing correlations in even-Z and odd-Z fissioning systems are very different; one is totally paired (the excitation energy at saddle is below the pairing gap) while the second has an ensemble of totally paired protons plus one unpaired proton; this additional proton is expected to weaken strongly the pairing correlations of the nucleus by blocking effects, while figure 4 shows that the pairing effects in close-by even- and odd$\mathrm{Z}$ fissioning systems have similar amplitudes. At higher excitation energy, the protonproton correlations in both systems are expected to be more similar, as the possible broken pairs in the even- and odd-Z systems equally weaken the pairing correlations of the remaining pairs. This would imply a smaller but similar even-odd effect in the fission of even and odd-Z nuclei at larger excitation energies. This is in contradiction with the data presented in figure 7 , which exhibit a systematically lower amplitude of 


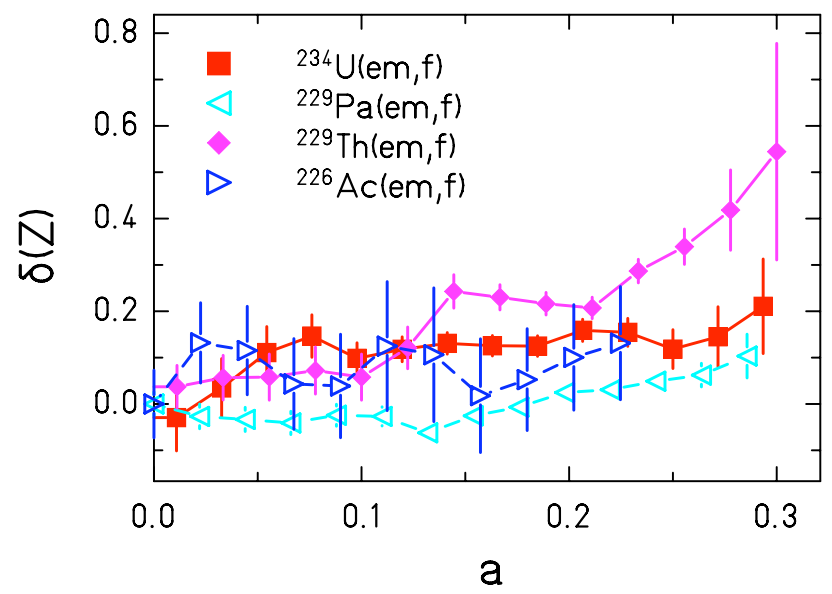

Figure 7. Local even-odd staggering in neighbouring even- $Z$ and odd-Z actinides measured in electromagnetic-induced fission [13]

the even-odd effect in odd-Z fissioning nuclei, and thus a larger influence of the unpaired proton on the magnitude of the even-odd effect at higher energies.

\section{Conclusion}

A systematic investigation on even-odd staggering in fission-fragment yields based on thermal-neutron-induced fission of actinides and electromagnetic-induced fission of neutron-deficient actinides is reported. This comprehensive data base allowed for the study of properties of the even-odd effect that were not accessible previously on the basis of thermal-neutron-induced fission alone due to the limited variety of fissioning nuclei investigated. The global even-odd effect is shown to vary with properties of the fissioning system, which do not have an evident connection with the available energy available on the deformation path from saddle to scission and the possible quasi-particle excitations. For all considered systems, the amplitude of the local even-odd effect at symmetry is shown to be small compared to the value at large asymmetries, while the latter is varying strongly with the fissioning system. The evolution of the global evenodd effect with the fissioning system is thus shown to be essentially due to the evolution of the fragment-distribution asymmetry, and its influence on the local even-odd effect. Consequently, the global even-odd effect cannot be considered to derive conclusions on dissipation, as it includes a strong contribution of the asymmetry of the fission-fragment distribution, which is unrelated with energy dissipation. The Coulomb parameter of the fissioning system, which is the conventional ordering parameter to investigate the evolution of the even-odd effect with the fissioning nucleus, is shown to be problematic. Despite its correlation to the available energy in the path down to scission, it is unable to describe the evolution of the even-odd effect over the ensemble of the investigated 
fissioning systems, which confirms that the even-odd effect is not only connected to the dissipated energy.

The results of the present analysis refute great part of the traditional theoretical models and ideas, which were developed to explain the even-odd effect in fission-fragment distributions. The new findings should be a guidance for a better understanding of this beautiful signature of the influence of nuclear structure on nuclear dynamics.

\section{Acknowledgments}

This work has been supported by the Région Basse Normandie with a Chair of Excellence position in GANIL, as well as by the EURATOM programme under the contract number 44816.

\section{References}

[1] Amiel S and Feldstein H 1975 Phys. Rev. C 11845

[2] Bocquet J P, Brissot R, Faust H R, Fowler M, Wilhelmy J, Asghar M and Djebara M 1990 Zeit. für Phys. A 33541

[3] Lang W, Clerc H-G, Wolfarth H, Schrader H and Schmidt K-H 1980 Nucl. Phys. A 34534

[4] Nifenecker H, Mariolopoulos G, Bocquet J P, Brissot R, Mme Hamelin Ch, Crancon J and Ristori Ch 1982 Zeit. für Phys. A 30839

[5] Bouzid B, Asghar M, Djebara M and Medkour M 1998 J. Phy. G: Nucl. Part. Phys. 241029

[6] Rejmund F, Ignatyuk A V, Junghans A R and Schmidt K-H 2000 Nucl. Phys. A 678215

[7] Schmidt K-H and Jurado B 2010 Phys. Rev. Let. 104212501

[8] Schmidt K-H and Jurado B 2010 arXiv:1007.0741v1 [nucl-th]

[9] Gönnenwein F 1991 The Nuclear Fission Process, Ed. C. Wagemans, CRC Press, London

[10] Bocquet J P and Brissot R 1989 Nucl. Phys. A 502 213c

[11] Naik H, Dange S P, Singh R J and Manohar S B 1997 Nucl. Phys. A 612143

[12] Unik J P, J E Gindler, Glendenin L E, Flynn K F, Gorski A and Sjoblom R K 1974 Proc. Symp. Phys. Chem. Fission, Rochester 1973, IAEA Vienna, 219

[13] Schmidt K-H et al. 2000 Nucl. Phys. A 665221

[14] Quade U et al. 1988 Nucl. Phys. A 4871

[15] Sida J L, Armbruster P, Bernas M, Bocquet J P, Brissot R and Faust H R 1989 Nucl. Phys. A $502233 \mathrm{c}$

[16] Schmitt C et al. 1984 Nucl. Phys. A 43021

[17] Rochman D, Faust H,Tsekhanovich I, Gönnenwein F, Storrer F, Oberstedt S and Sokolov V 2002 Nucl. Phys. A 7103

[18] Rochman D, Tsekhanovich I, Gönnenwein F, Sokolov V, Storrer F, Simpson G and Serot O 2004 Nucl. Phys. A 7353

[19] Djebara M et al 1989 Nucl. Phys. A 496346

[20] Hentzschel R, Faust H R, Denschlag H O, Wilkins B D and Gindler J 1994 Nucl. Phys. A 571427

[21] Rejmund F, Caamaño M and Schmidt K-H 2010, GANIL P 2010-01, hal.in2p3.fr:in2p3-00487186

[22] Böckstiegel C, Steinhäuser S, Schmidt K-H, Clerc H-G, Grewe A, Heinz A, de Jong M, Junghans A R, Müller J and Voss B 2008 Nucl. Phys. A 80212

[23] Pommé S, Jacobs E, Persyn K, De Frenne D, Govaert K and Yoneama M L 1993 Nucl. Phys. A $\mathbf{5 6 0} 689$

[24] Persyn K, Jacobs E, Pommé S, De Frenne D, Govaert K and Yoneama M L 1997 Nucl. Phys. A 620171 
[25] Tracy B L, Chaumont J, Klapisch R, Nitschke J M, Poskanzer A M, Roeckl E and Thibault C 1972 Phys. Rev. C 5222

[26] Tsekhanovich I, Denschlag H-O, Davi M, Büyükmumcu Z, Gönnenwein F, Oberstedt S and Faust H R 2001 Nucl. Phys. A 688633

[27] Tsekhanovich I, Denschlag H-O, Davi M, Büyükmumcu Z, Woestheinrich M, Gönnenwein F, Oberstedt S and Faust H R 1999 Nucl. Phys. A 658217

[28] Steinhäuser S et al. 1998 Nucl. Phys. A 63489

[29] Wahl A C 1985 Phys. Rev. C 32184 